\title{
AC-field Induced Gap Opening in the Vicinity of Extra Dirac Points in Band Structure of Graphene Superlattice
}

\author{
S.V. Kryuchkov $1,2,{ }^{*}$, E.I. Kukhar' ${ }^{\prime 1}{ }^{+}$ \\ ${ }^{1}$ Volgograd State Socio-Pedagogical University, Physical Laboratory of Low-Dimensional Systems, 27, V.I. Lenin \\ ave, Volgograd 400066, Russia \\ ${ }^{2}$ Volgograd State Technical University, 28, V.I. Lenin ave., Volgograd 400005, Russia
}

(Received 07 December 2016; published online 23 December 2016)

\begin{abstract}
Floquet spectrum of electrons in graphene superlattice subjected to the ac electric field was investigated. The ac-field vector was assumed to oscillate perpendicularly to the superlattice axis. In the vicinity of extra Dirac points of superlattice band structure the gap was shown to be dynamically induced by the acfield. Such effects are due to the nonadditivity of the electron spectrum of graphene superlattice.
\end{abstract}

Keywords: Graphene Superlattice, Extra Dirac Points, Floquet Spectrum.

DOI: $10.21272 /$ jnep.8(4(2)).04057

PACS numbers: 73.21.Cd, 72.20.Ht, 73.50.Fq

\section{INTRODUCTION}

Development of the technologies of fabrication of graphene-like materials [1, 2] stimulates investigations in the area of 2D Dirac-like fermions [3, 4]. Presently graphene is offered to be the basis of the modern nanoelectronic devices [5-8]. From an applicationtechnological point of view the tunability of electronic and optical properties of graphene materials by the external electromagnetic (EM) fields has particular importance. This gives the way of manipulation of spectrum parameters without interfering in the internal structure of graphene material [9-11].

High-frequency EM radiation is known to lead to the dynamical renormalization of the electron spectrum of low-dimensional systems [12]. Induced by electronphoton coupling modification of band structure of graphene based materials is one of the subjects of intense activity last time $[13,14]$. Some materials (including graphene) can be tuned by time-dependent driving (further it is called as ac-driving) into specific states called as the Floquet topological insulators [16]. Laserinduced gap in the vicinity of the Dirac points of the band structure of the originally gapless graphene layer had been predicted in [13]. Theory describing magnetoelectronic properties of ac-driven graphene was developed in [17-19], where the possibility of Landau levels manipulation by ac electric field effect was predicted. Moreover, there are the experiments [20,21] which showed the possibility of Floquet topological insulators.

Such results are important for the engineering of graphene based devices with tunable characteristics. The high mobility of electrons in graphene, on the one hand, and the possibility of using of superlattices (SL) as a working medium of amplifiers of EM radiation and sources of solitary EM waves [22], on the other hand, make graphene SL (GSL) attractive to researchers $[23,24]$. And investigations of ac-field modification of band structure are of interest not only for graphene but for GSL also. Thus there is the problem of the ac electric field effect on the electron spectrum of GSL. Below we consider this question, find the Floquet spectrum (quasienergy) of ac-driven GSL within non-resonant approximation and predict the ac-field induced gaps in the vicinity of so-called extra Dirac points in band structure of GSL.

\section{EXTRA DIRAC POINTS}

A number of investigations had proposed various ways of GSL fabrication [25-28]. In [29] the next Hamiltonian had been offered to describe the electron states in GSL in the vicinity of Dirac point: $\hat{H}(\mathbf{r}, t)=v_{\mathrm{F}} \hat{\boldsymbol{\sigma}} \cdot \hat{\mathbf{p}}+V(x)$. Here graphene is assumed to be in the plane $x y, \hat{\boldsymbol{\sigma}}=\left(\hat{\sigma}_{x}, \hat{\sigma}_{y}\right)$, are the Pauli matrixes, $v_{\mathrm{F}}$ is the Fermi velocity, $V(x)$ is the additional potential periodical along the axis $O x$ : $V(x+d)=V(x), d$ is the GSL period.

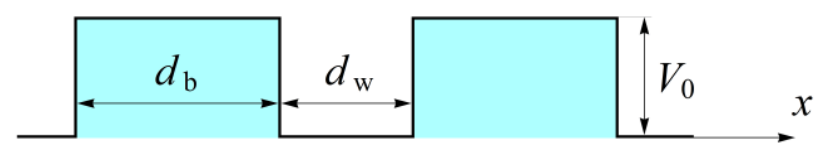

Fig. 1 - Spatial periodic modulation of the band gap in graphene

Note that in [29] the Kronig-Penney model was used for the potential $V(x)$. Within this model the emergence of so-called extra Dirac points in the band structure of the GSL had been predicted in [29]. The ac-field was absent in the problem investigated in [29]. Apart from the usual Dirac point $\left(p_{x}=p_{y}=0\right)$ the extra Dirac points are arranged along the $p_{y}$ axis and their location is determined by the relations [29] $(\hbar=1)$ :

$$
v_{\mathrm{F}}^{2} p_{y, n}^{2}=\frac{V_{0}^{2}}{4}-\frac{4 \pi^{2} v_{\mathrm{F}}^{2} n^{2}}{d^{2}}
$$

\footnotetext{
*svkruchkov@yandex.ru

+ eikuhar@yandex.ru
} 
where $n$ is nonzero integer, $V_{0}$ is the barrier height, well and barrier widths are assumed to be equal. Indeed, the conduction and valence bands meet at points with $p_{x}=0$ and $p_{y}=p_{y, n}$, wherein energy is $\varepsilon=V_{0} / 2$.

\section{AC-DRIVEN GSL}

Now GSL is suggested to be subjected to the timeperiodical electric field with intensity oscillating in the plane $x y$. This can be achieved by placing of the GSL in the field of EM wave which propagates perpendicular to the plane $x y$ so the vector of electric field oscillates in $x y$ and is described by the time-dependent vector potential $\mathbf{A}^{\mathrm{ac}}(t)=\mathbf{A}^{\mathrm{ac}}(t+T)$, where $T$ is the period of ac-field. The quantum mechanical state of the electron is described by a spinor $\psi$ which in the vicinity of Dirac point $\mathrm{K}$ obeys the Dirac-like equation $i \partial_{t} \psi=\hat{H}_{t} \psi$. Here

$$
\hat{H}_{t}(\mathbf{r}, t)=v_{\mathrm{F}}\left[\mathbf{p}+e \mathbf{A}^{\mathrm{ac}}(t)\right] \cdot \hat{\boldsymbol{\sigma}}+V(x),
$$

spinor $\psi$ has two components corresponding to the different values of pseudospin denoting the graphene sublattice $(c=1)$ and obeys the Floquet theorem:

$$
\psi(\mathbf{r}, t)=u(\mathbf{r}, t) e^{-i \varepsilon_{\mathrm{eff}} t}
$$

where $\varepsilon_{\text {eff }}$ is quasienergy, $u(\mathbf{r}, t)$ is the spinor with components which are time-periodic functions with period $T$. The action of the operator (1) on the function (2) leads to the equation

$$
\left[\hat{H}_{t}(\mathbf{r}, t)-i \frac{\partial}{\partial t}\right] u=\varepsilon_{\text {eff }} u .
$$

Polarization of the EM wave is assumed to be linear. The direction of the ac electric field vector is proposed to oscillate perpendicularly to the SL axis $\left(A_{x}^{\mathrm{ac}}=0\right)$. To reduce the time-dependent problem described by equation (3) to a stationary problem the spinor $u$ is written in the form $u(\mathbf{r}, t)=\hat{U} \chi(\mathbf{r}, t)$. Here $\hat{U}$ is the unitary operator which is equal

$$
\hat{U}=e^{-i a_{y} \hat{\sigma}_{y}}, a_{y}=e v_{\mathrm{F}} \int A_{y}^{\mathrm{ac}}(t) \mathrm{d} t .
$$

After use of the unitary transformation provided by the operator (4) we arrive at the next equation

$$
\left[-i \frac{\partial}{\partial t}+v_{\mathrm{F}} \hat{p}_{x} \hat{\sigma}_{x} \hat{U}^{2}+v_{\mathrm{F}} \hat{p}_{y} \hat{\sigma}_{y}+V(x)\right] \chi=\varepsilon_{\text {eff }} \chi .
$$

Further to find the quasienegry $\varepsilon_{\text {eff }}$ appearing in (5) we use the averaging method [30]. To this end we represent the spinor $\chi$ and time-periodical operator $\hat{U}^{2}$ as the sums each contains the constant term and highfrequency term: $\chi(t)=\chi_{0}+\chi_{\mathrm{ac}}(t), \hat{U}^{2}(t)=\hat{\gamma}_{0}+\hat{U}_{\mathrm{ac}}^{2}(t)$, where

$$
\begin{gathered}
\chi_{0}=\left(\begin{array}{c}
b_{0}^{\uparrow} \\
b_{0}^{\downarrow}
\end{array}\right), \chi_{\mathrm{ac}}(t)=\sum_{k \neq 0}\left(\begin{array}{c}
b_{k}^{\uparrow} \\
b_{k}^{\downarrow}
\end{array}\right) e^{i k \omega t}, \hat{U}_{\mathrm{ac}}^{2}(t)=\sum_{k \neq 0} \hat{\gamma}_{k} e^{i k \omega t}, \\
\omega=\frac{2 \pi}{T}, \\
\hat{\gamma}_{k}=\frac{1}{2 \pi} \int_{-\pi}^{+\pi} \hat{U}^{2}(\xi) e^{-i k \xi} \mathrm{d} \xi
\end{gathered}
$$

So that the average values are: $\langle\chi\rangle=\chi_{0},\left\langle\chi_{\mathrm{ac}}\right\rangle=0$, $\left\langle\hat{U}^{2}\right\rangle=\hat{\gamma}_{0}$ and $\left\langle\hat{U}_{\mathrm{ac}}^{2}\right\rangle=0$. To neglect the high-frequency part of the equation (5) the next conditions are supposed to be performed

$$
\sum_{k \neq 0}\left|b_{k}^{\uparrow, \downarrow}\right|^{2}<<\left|b_{0}^{\uparrow, \downarrow}\right|^{2}
$$

After averaging in the both parts of the equation (5) one can find the relation for the constant part

$$
\left(\hat{H}_{0}-\varepsilon_{\text {eff }}\right) \chi_{0}=v_{\mathrm{F}} \hat{p}_{x} \hat{\sigma}_{x} \sum_{k \neq 0} \hat{\gamma}_{k}\left\langle\chi_{\mathrm{ac}} e^{i k \omega t}\right\rangle
$$

where

$$
\hat{H}_{0}=v_{\mathrm{F}} \hat{p}_{x} \hat{\sigma}_{x} \hat{\gamma}_{0}+v_{\mathrm{F}} \hat{p}_{y} \hat{\sigma}_{y}+V(x) .
$$

Now we leave only the oscillating terms in (5). In which connection the conditions (7) allow us to neglect the terms containing $\chi_{\mathrm{ac}}$ and to arrive at the expression containing only terms with $\chi_{0}$ and with $\partial_{t} \chi_{\mathrm{ac}}$. After integrating of this expression we obtain

$$
\chi_{\mathrm{ac}}(t)=-v_{\mathrm{F}} \hat{p}_{x} \hat{\sigma}_{x} \sum_{k \neq 0} \frac{\hat{\gamma}_{k} \chi_{0}}{k \omega} e^{i k \omega t}
$$

After substitution of (10) into (8) we find that the right side of (8) is zero. As a result the problem of the electron state in ac-driven GSL is reduced to the solving of the equation

$$
\hat{H}_{0} \chi_{0}=\varepsilon_{\text {eff }} \chi_{0} .
$$

For the sinusoidal radiation with amplitude of electric field intensity $E_{0}$ and frequency $\omega$ formula (9) gives $\quad \hat{H}_{0}=u_{\mathrm{F}} \hat{p}_{x} \hat{\sigma}_{x}+v_{\mathrm{F}} \hat{p}_{y} \hat{\sigma}_{y}+V(x), \quad$ where $u_{\mathrm{F}}=v_{\mathrm{F}} J_{0}\left(2 a_{0}\right)$ is the Fermi velocity renormalized by the ac-field effect, $a_{0}=e v_{\mathrm{F}} E_{0} / \omega^{2}$ is the dimensionalless amplitude of ac-field, $J_{k}(\xi)$ is the Bessel function of integer order. The approximation provided by the conditions (7) is seen from the formula (10) to work correctly if the next inequality is performed

$$
v_{\mathrm{F}}^{2} p_{x}^{2} \sum_{k=1}^{\infty} \frac{J_{k}^{2}\left(2 a_{0}\right)}{k^{2} \omega^{2}}<<1 .
$$

Also the parameter $2 a_{0}$ should be far from the zeros of the Bessel function $J_{0}\left(2 a_{0}\right)$. 

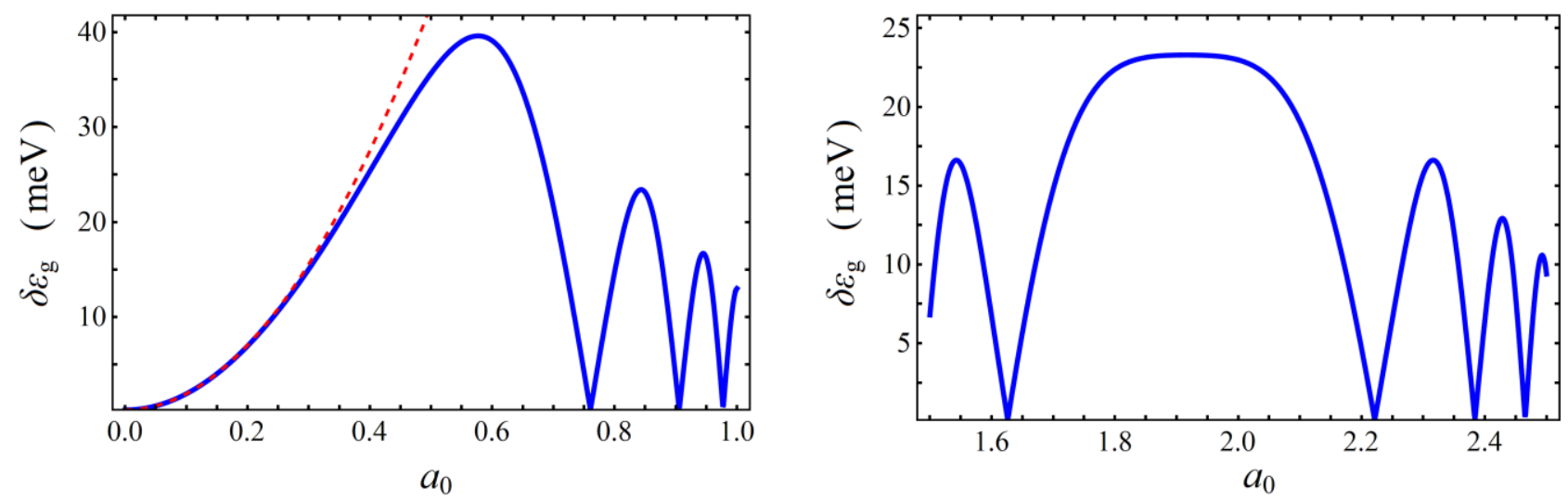

Fig. 2 - Dependences of the gap induced dynamically by the ac-field in the vicinity of the extra Dirac points with $n= \pm 1$ on the amplitude $V_{0}=0.45 \mathrm{eV}$, dashed line corresponds to the approximate formula (15)

\section{AC-FIELD INDUCED GAP OPENING IN THE VICINITY OF EXTRA DIRAC POINTS}

Here we consider the function $V(x)$ written in the form of square-wave barriers as it is shown in Fig. 1. The widths of well and barrier are defined as $d_{\mathrm{w}}$ and $d_{\mathrm{b}}$ correspondingly:

$$
V(x)=\left\{\begin{array}{l}
0, \quad(n-1) d<x<d_{\mathrm{w}}+(n-1) d, \\
V_{0}, \quad d_{\mathrm{w}}+(n-1) d<x<n d,
\end{array}\right.
$$

$d=d_{\mathrm{w}}+d_{\mathrm{b}}$. The parts of the wave functions defined as $\chi_{n}^{\mathrm{w}, \mathrm{b}}(x)$ correspond to the electron states in the well and barrier with number $n$ and satisfy the boundary conditions

$$
\chi_{n}^{\mathrm{w}}\left(d_{\mathrm{w}}-0\right)=\chi_{n}^{\mathrm{b}}\left(d_{\mathrm{w}}+0\right), \chi_{n}^{\mathrm{b}}(d-0)=\chi_{n+1}^{\mathrm{w}}(+0) .
$$

Dispersion relation in this case is derived from the equation $\operatorname{Re} T_{11}=\cos \left(p_{x} d\right)$, where $T_{11}$ is the transfer matrix element located in the first row and first column. Transfer matrix can be written as

$$
\hat{T}=\hat{\Omega}_{1}^{-1}(0) \hat{\Omega}_{2}(d) \hat{\Omega}_{2}^{-1}\left(d_{\mathrm{w}}\right) \hat{\Omega}_{1}\left(d_{\mathrm{w}}\right),
$$

where

$$
\begin{aligned}
\hat{\Omega}_{j}(x)= & \left(\begin{array}{cc}
1 & 1 \\
\beta_{j} & -\beta_{j}^{*}
\end{array}\right) e^{i \hat{\sigma}_{z} p_{j} x}, \beta_{j}=\frac{u_{\mathrm{F}} p_{j}+i v_{\mathrm{F}} p_{y}}{\varepsilon_{\mathrm{eff}}-V_{0} \delta_{j, 2}}, \\
& u_{\mathrm{F}}^{2} p_{j}^{2}=\left(\varepsilon_{\mathrm{eff}}-V_{0} \delta_{j, 2}\right)^{2}-v_{\mathrm{F}}^{2} p_{y}^{2},
\end{aligned}
$$

$\delta_{k, j}$ is Kronecker symbol, $j=1,2$. Having found the real part of $T_{11}$ we obtain

$$
\begin{aligned}
& -\frac{u_{\mathrm{F}}^{2}\left(p_{1}^{2}+p_{2}^{2}\right)-V_{0}^{2}}{2 u_{\mathrm{F}}^{2} p_{1} p_{2}} \sin \left(p_{1} d_{\mathrm{w}}\right) \sin \left(p_{2} d_{\mathrm{b}}\right)+ \\
& +\cos \left(p_{1} d_{\mathrm{w}}\right) \cos \left(p_{2} d_{\mathrm{b}}\right)=\cos \left(p_{x} d\right)
\end{aligned}
$$

If ac-field is absent $\left(a_{0}=0\right)$ formula (14) yields the result [29].
It is follows from the dispersion relation (14) that electron states in the extra Dirac points (i.e. states with $p_{x}=0$ and $\left.p_{y}=p_{y, n}\right)$ do not exist. It means the appearance of the gaps $\delta \varepsilon_{\mathrm{g}}$ which are dynamically induced by ac-field in the vicinity of each extra Dirac points said above. In the case of weak ac-field $\left(a_{0}<<1\right)$ the calculations give the next formula for the gap width

$$
\delta \varepsilon_{\mathrm{g}} \sim \frac{32 \pi^{2} v_{\mathrm{F}}^{2} n^{2}}{V_{0}^{2} d} v_{\mathrm{F}}\left|p_{y, n}\right| a_{0}^{2} .
$$

Predicted in this section dynamical appearance of the gap in the vicinity of extra Dirac points of the band structure of GSL due to the ac-field is new result. Dependences of $\delta \varepsilon_{\mathrm{g}}$ on the ac-field amplitude calculated within the Kronig-Penney model are shown in Fig. 2, where dashed line corresponds to the approximate formula (15) for the next parameters values: $v_{\mathrm{F}}=10^{8}$ $\mathrm{cm} / \mathrm{s}, d=2 \cdot 10^{-6} \mathrm{~cm}, d_{\mathrm{b}}=d_{\mathrm{w}}$.

Note that described modification of the electron spectrum is owing to the effect of ac-field polarized perpendicularly to the GSL axis. Such effect is possible due to the nonadditivity of the dispersion law of the GSL.

\section{CONCLUSION}

Above the Floquet spectrum of electrons in GSL subjected to the ac electric field has been calculated with the help of the averaging method. The calculations have been performed within the Kronig-Penney model. Nonadditivity of GSL electron spectrum has been shown to lead to the dynamical modification of the dispersion law of electrons even if the vector of ac electric field is applied perpendicularly to the GSL axis. Such feature makes GSL different from the conventional SL with additive spectrum where the effect of acfield with such polarization leads only to a shift of spectrum as a whole.

We have found that in the vicinity of extra Dirac points of GSL band structure the gap $\delta \varepsilon_{\mathrm{g}}$ is dynamically induced by the ac-field effect. For weak ac-fields this 
gap increases as $a_{0}^{2}$. It is quite seen from the Fig. 2 (the region of dashed line). For arbitrary values of amplitude $a_{0}$ except the vicinity of the zeros of Bessel function $\left(J_{0}\left(2 a_{0}\right) \neq 0\right)$ the gap $\delta \varepsilon_{\mathrm{g}}$ oscillates with $a_{0}$.

At the end we make numerical estimates. Dynamical gap is seen from the Fig. 2 to open and reach its maximum $\delta \varepsilon_{\mathrm{g}}=40 \mathrm{meV}$ for the intensity of $I=2 \mathrm{~W} / \mathrm{mm}^{2}$ in the case when radiation is polarized

\section{REFERENCES}

1. P. Avouris, C. Dimitrakopoulos, Mater. Today 15, 86 (2012).

2. M. Hofmann, W.-Y. Chiang, T.D. Nguyn, Y.-P. Hsieh, Nanotechnology 26, 335607 (2015).

3. H. Fukuyama, Y. Fuseya, M. Ogata, A. Kobayashi, Y. Suzumura, Physica B 407, 1943 (2012).

4. V.M. Apalkov, T. Chakraborty, Phys. Rev. Lett. 112, 176401 (2014).

5. S.V. Morozov, K.S. Novoselov, M.I. Katsnelson, F. Schedin, D.C. Elias, J.A. Jaszczak, A.K. Geim, Phys. Rev. Lett. 100, 016602 (2008).

6. D.A. Svintsov, V.V. Vyurkov, V.F. Lukichev, A.A. Orlikovsky, A. Burenkov, R. Oechsner, Semiconductors 47, 279 (2013).

7. A.S. Moskalenko, S.A. Mikhailov, J. Appl. Phys. 115, 203110 (2014).

8. S. Sekwao, J.-P. Leburton, Appl. Phys. Lett. 106, 063109 (2015).

9. Yu.E. Lozovik, A.A. Sokolik, Nanoscale Res. Lett. 7, 134 (2012).

10. H.K. Kelardeh, V. Apalkov, M.I. Stockman, Phys. Rev. B 90, 085313 (2014).

11. E. Margapoti，P. Strobel，M.M. Asmar，M. Seifert，J. Li, M. Sachsenhauser, Ö. Ceylan, C.-A. Palma, J.V. Barth, J.A. Garrido, A. Cattani-Scholz, S.E. Ulloa, J.J. Finley, Nano Lett. 14, 6823 (2014).

12. M. Holthause, D.W. Hone, Phys. Rev. B 49, 16605 (1994).

13. T. Oka, H. Aoki, Phys. Rev. B 79, 081406(R) (2009).

14. G. Usaj, P.M. Perez-Piskunow, L.E.F. Foa Torres, C.A. Balseiro, Phys. Rev. B 90, 115423 (2014).

15. N.H. Lindner, G. Refael, V. Galitski, Nat. Phys. 7, 490 (2011). perpendicularly to the GSL axis and have the frequency $\omega=10 \mathrm{THz}$. So such dynamical gap can be measured experimentally.

\section{ACKNOWLEDGEMENTS}

The work was supported with the funding of the Ministry of Education and Science of the Russian Federation within the base part of the State task: Code: 3.2797.2017/PCh.

16. S.V. Kryuchkov, E.I. Kukhar', Physica B 445, 93 (2014).

17. K. Kristinsson, O.V. Kibis, S. Morina, I.A. Shelykh, Sci. Rep. 6, 20082 (2016).

18. O.V. Kibis, S. Morina, K. Dini, I.A. Shelykh, Phys. Rev. B 93, 115420 (2016).

19. M.C. Rechtsman, J.M. Zeuner, Y. Plotnik, Y. Lumer, D. Podolsky, F. Dreisow, S. Nolte, M. Segev, A. Szameit, Nature 496, 196 (2013).

20. Y.H. Wang, H. Steinberg, P. Jarillo-Herrero, N. Gedik, Science 342, 453 (2013).

21. A.V. Shorokhov, M.A. Pyataev, N.N. Khvastunov, T. Hyart, F.V. Kusmartsev, K.N. Alekseev, JETP Lett. 100, 766 (2015).

22. R.V. Gorbachev, J.C.W. Song, G.L. Yu, A.V. Kretinin, F. Withers, Y. Cao, A. Mishchenko, I.V. Grigorieva, K.S. Novoselov, L.S. Levitov, A.K. Geim, Science 346, 448 (2014).

23. H. Cheng, C. Li, T. Ma, L.-G. Wang, Y. Song, H.-Q. Lin, Appl. Phys. Lett. 105, 072103 (2014).

24. L.A. Chernozatonskii, P.B. Sorokin, E.E. Belova, I. Bryuning, A.S. Fedorov, JETP Lett. 85, 77 (2007).

25. A.L. Vazquez de Parga, F. Calleja, B. Borca, M.C.G. Passeggi, J.J. Hinarejos, F. Guinea, R. Miranda, Phys. Rev. Lett. 100, 056807 (2008).

26. H. Sevinçli, M. Topsakal, S. Ciraci, Phys. Rev. B 78, 245402 (2008).

27. P.V. Ratnikov, A.P. Silin, JETP Lett. 100, 311 (2014).

28. M. Barbier, P. Vasilopoulos, F.M. Peeters, Phys. Rev. B 81, 075438 (2010).

29. N.N. Bogoliubov, Y.A. Mitropolsky, Asymptotic Methods in the Theory of Non-Linear Oscillations (New York: Gordon and Breach: 1961). 